\title{
Nivel de depresión del adulto mayor que asiste a un Centro Gerontológico
}

Depression Level of the Elderly who attends a Gerontological Center

\author{
Naara N. Chavez-Castillo ${ }^{a}$, Karen A. Jiménez-Sánchez ${ }^{b}$,Ariadna I. Téllez-Cruz ${ }^{c}$, Gabriela
}

Maldonado-Muñiz ${ }^{d}$, Edith A. Cano-Estrada ${ }^{e}$, Gustavo Vélez-Díaz ${ }^{f}$

\begin{abstract}
:
Introduction: Depression is a frequent disorder in older adults and according to the World Health Organization, which by 2020 will be the second cause of disability worldwide. It can usually manifest as sadness. Objective: To identify the level of depression of the older adult person who attends a Gerontological Center. Method: Quantitative, descriptive study, 56 people over 60 were included, from a Gerontological Center; a sociodemographic data card and the Yesavage Geriatric Depression Scale with a reliability of Cronbach's Alpha of .87 were applied. Results: $n=56,71.4 \%$ were women, with an average age of $71.46 \pm 7.483$ years; $69.6 \%$ of older adults found normal depression, $26.8 \%$ and 3.6\%. Discussion: In the study entitled "Effect of occupational therapy in the elderly with depression", conducted by López and Colombia in 2017 with a total of 31 older adults, it is said that the majority were women with $67.7 \%$. Finding similarity to his study where $83.9 \%$ does not present depression. Conclusions: In the present study it was found that according to the results that $69.6 \%$ of older adults were found with normal depression.
\end{abstract}

Keywords:

Depression, Elderly, Sadness.

\section{Resumen:}

Introducción: La depresión es un trastorno frecuente en los adultos mayores y según la Organización Mundial de la Salud afirma que para el 2020 será la segunda causa de discapacidad a nivel mundial. Generalmente puede manifestarse como tristeza. Objetivo: Identificar el nivel de depresión del adulto mayor que asiste a un Centro Gerontológico. Método: Estudio cuantitativo, descriptivo, se incluyeron 56 personas mayores de 60 años, de un Centro Gerontológico; se aplicó una cédula de datos sociodemográficos y la Escala de Depresión Geriátrica de Yesavage con una fiabilidad de Alfa de Cronbach de .87. Resultados: n=56, $71.4 \%$ eran mujeres, con media de edad de $71.46 \pm 7.483$ años; el $69.6 \%$ de los adultos mayores se encontraron con depresión normal, un $26.8 \%$ leve y $3.6 \%$ severa. Discusión: En el estudio titulado "Efecto de la terapia ocupacional en el adulto mayor con depresión" realizado por López y cols en el 2017 con un total de 31 Adultos Mayores, se observó que la mayoría eran mujeres con 67.7\%. Encontrando similitud con su estudio donde el $83.9 \%$ no presenta depresión. Conclusiones: En el presente estudio se encontró que de acuerdo con los resultados que le $69.6 \%$ de los adultos mayores se encontraron con depresión normal.

\section{Palabras Clave:}

Depresión, Adulto Mayor, Tristeza.

\footnotetext{
a Alumno de la Universidad Autónoma del Estado de Hidalgo, Escuela Superior de Tlahuelilpan. E-mail: ch265358@uaeh.edu.mx

b Alumno de la Universidad Autónoma del Estado de Hidalgo, Escuela Superior de Tlahuelilpan. E-mail: ji294254@uaeh.edu.mx c Alumno de la Universidad Autónoma del Estado de Hidalgo, Escuela Superior de Tlahuelilpan. E-mail: te347891@uaeh.edu.mx

d Autor de Correspondencia, Profesor Investigador de la Universidad Autónoma del Estado de Hidalgo, Escuela Superior de Tlahuelilpan. ORCID: https://orcid.org/0000-0002-4967-1812.E-mail: gmaldonado@uaeh.edu.mx

e Profesor Investigador de la Universidad Autónoma del Estado de Hidalgo, Escuela Superior de Tlahuelilpan. ORCID: https://orcid.org/00000002-8315-1087.E-mail: edith_cano@uaeh.edu.mx

${ }_{\mathrm{f}}^{\mathrm{f}}$ Profesor Investigador de la Universidad Autónoma del Estado de Hidalgo, Escuela Superior de Tlahuelilpan. E-mail:
} 


\section{Introducción}

Actualmente, por primera vez en la historia, la mayor parte de la población tiene una esperanza de vida igual o superior a los 60 años. Para 2050, se espera que la población mundial en esa franja de edad llegue a los 2000 millones, un aumento de 900 millones con respecto a 2015 (OMS, 2018). De acuerdo con el Consejo Nacional de Población (CONAPO) en 2017 residen en el país 12, 973,411 personas de 60 y más años de los cuales 53.9\% son mujeres, y $46.1 \%$ son hombres (CNDH, 2017).

El envejecimiento es un proceso inherente a la vida humana, el cual constituye una experiencia única, heterogénea y su significado varía dependiendo de la connotación que cada cultura haga de él. Se entiende como un fenómeno multidimensional que involucra todas las dimensiones de la vida humana (Alvarado-García, et al., 2014). Es una etapa de pérdidas, como la vitalidad, cambio en su ritmo de vida, disminución de sus funciones y capacidades, de figuras significativas e importantes, como gente de su edad, de su pareja, la separación de los hijos y las familias, el inicio de una serie de enfermedades crónico degenerativas, merma económica, lo que puede llevar al adulto mayor a la depresión (IMSS, 2014).

La depresión es una enfermedad mental que afecta a más de 350 millones de personas en todo el mundo, siendo los Adultos Mayores uno de los grupos más vulnerables (IMSS, 2014).

La depresión es un trastorno mental frecuente que se caracteriza por la presencia de tristeza, perdida de interés o placer, sentimientos de culpa o falta de autoestima trastorno del sueño o de apetito, sensación de cansancio y o falta de concentración muchas veces no tomamos en cuenta los cambios psicológicos que sufre el adulto mayor y estos van a influir en su estado de ánimo y en su conducta de manera positiva o negativa todo depende del entorno familiar y cultural (OMS, 2017).

El presente trabajo de investigación tuvo el objetivo de Identificar el nivel de depresión del adulto mayor que asiste a un Centro Gerontológico.

\section{Planteamiento del problema o exposición del caso}

En la etapa del adulto mayor, se producen cambios graduales y progresivos que con el paso del tiempo llevan a la persona al final de la vida, el bienestar durante este periodo es directamente proporcional con el estilo de vida que la persona haya llevado, la presencia o no de patologías y de cómo el individuo afronte el envejecimiento desde el punto de vista psicológico (Durán-Badillo, et al., 2013)

La Organización Mundial de la Salud (OMS) afirma que actualmente cinco de las 10 causas más importantes de discapacidad son de origen psiquiátrico y se piensa que para el 2020 la depresión será la segunda causa de discapacidad a nivel mundial (OMS, 2017).

La depresión contribuye a elevar la morbi-mortalidad, disminuye la calidad de vida e incrementa costos del cuidado de la salud. A pesar de una mayor conciencia pública de la depresión y una gran variedad de tratamientos eficaces la depresión en los adultos mayores sigue siendo poco reconocida y mucho menos tratada. Es considerada una de las causas prevenibles y tratables del envejecimiento patológico (Secretaría de Salud, 2011).

Como consecuencia se puede observar la disminución de energía, merma en sus actividades, disfunción social, familiar y en caso extremo el suicidio. En adultos después de los 65 años hay un alto índice de suicidios (IMSS, 2014).

\section{Conocimiento previo}

Acosta y cols., en el estudio titulado "Prevalencia de depresión en el adulto mayor en los centros de vida en Cartagena año 2015", el cual tiene como objetivo determinar la prevalencia de depresión en el adulto mayor en los centros de vida en Cartagena, con enfoque cuantitativo, en 398 adultos mayores inscritos en los 16 centros de vida de Cartagena, utilizaron la Escala de Depresión Geriátrica Yesavage (Geriatric Depression Scale), GDS-15, dando como resultado que la prevalencia de depresión fue de $7.7 \%$ en los adultos mayores institucionalizados; el 6.7\% presentaron depresión leve, el $1 \%$ depresión severa y el $92.2 \%$ de los adultos mayores no presentaron depresión (Acosta-López, 2016).

\section{Metodología de diseño o de estudio}

Estudio con enfoque cuantitativo, descriptivo, universo de 56 adultos mayores en un periodo de junio-diciembre 2018. El muestreo fue por disponibilidad, incluyendo a los adultos mayores inscritos al Centro Gerontológico Integral de Tlahuelilpan, Hidalgo que aceptaron participar. No se encontraron adultos mayores con tratamiento psicológico, o con discapacidad auditiva, por lo que no se aplicaron los criterios de exclusión.

Se utilizó la Escala de Depresión Geriátrica (GDS) de Yesavage diseñada para medir el nivel de depresión en el adulto mayor, el instrumento consta de 15 ítems, posee una estructura cerrada tipo dicotómica. Considerando para su calificación un rango de 0-5 como normal, de 6-10 deprimido leve y con una puntuación mayor a 10 se considera como depresión severa. Este instrumento es aplicado en la población mexicana por la Secretaria de Salud, con una fiabilidad de Alfa de Cronbach de .87.

Apegado a la declaración de Helsinki y a la Ley General de Salud se firmó el consentimiento informado previo a aplicación de instrumento, respetando los derechos del Adulto mayor para que en cualquier momento, estudio considerado de riesgo mínimo.

Análisis: se realizó una base de datos en el Statistical Package for the Social Sciences (SPSS) versión 21.

\section{V.Aplicación}

Desde un punto de vista biológico, el envejecimiento es la consecuencia de la acumulación de una gran variedad de daños moleculares y celulares a lo largo del tiempo, lo que lleva a un descenso gradual de las capacidades físicas y mentales, un aumento del riesgo de enfermedad, y finalmente a la muerte. Ahora bien, esos cambios no son 
lineales ni uniformes, y su vinculación con la edad de una persona en años es más bien relativa. Si bien algunos septuagenarios disfrutan de una excelente salud y se desenvuelven perfectamente, otros son frágiles y necesitan ayuda considerable. Además de los cambios biológicos, el envejecimiento también está asociado con otras transiciones de la vida como la jubilación, el traslado a viviendas más apropiadas, y la muerte de amigos y pareja (OMS, 2018)

Para otorgar el cuidado de enfermería es necesario valorar de forma holística al adulto mayor, y determinar si un estado de depresión puede estar afectando su situación de salud, por lo que la aplicación de una escala validad y con confiabilidad es de gran utilidad.

\section{Resultados}

$\mathrm{n}=56$ adultos mayores, $28.6 \%$ de los adultos mayores eran de género masculino y $71.4 \%$ eran femenino, $87.5 \%$ de los adultos mayores contaban con escolaridad de nivel básico y $3.6 \%$ con nivel superior, $69.6 \%$ estaban casados, $69.6 \%$ se ocuparon del hogar, $53.6 \%$ contaban con beneficio de gobierno de 65 y más, $48.2 \%$ eran afiliados por el seguro popular y $9.1 \%$ no tenían seguridad social. $66.1 \%$ vivían con su pareja, $23.2 \%$ con hijos y $10.7 \%$ solos. El $92.9 \%$ tenían casa propia y un $7.1 \%$ no tenían casa propia. $62.5 \%$ padecían hipertensión y diabetes. Ver Tabla 1

Nivel de depresión: $69.6 \%(n=39)$ de los adultos mayores tenían nivel normal, $26.8 \%(n=15)$ leve y $3.6 \% \quad(n=2)$ severa. Ver tabla 2.

\section{Discusión}

En el presente estudio titulado "Nivel de depresión del adulto mayor que asiste a un Centro Gerontológico" se encontró que en cuanto al perfil demográfico $71.4 \%$ eran mujeres y un $28.6 \%$ hombres, lo cual tiene similitud de acuerdo con el Consejo Nacional de Población (CONAPO) en 2017 residen en el país 12973411 personas de 60 y más años de los cuales $53.9 \%$ son mujeres, y $46.1 \%$ son hombres (CNDH, 2017), se infiere que asisten más mujeres al centro Gerontológico Integral por que las actividades que ahí se realizan son de preferencia de las mujeres ya que se imparten talleres de cocina, tejido, manualidades y Tai Chi.

Asimismo, en el estudio titulado "Efecto de la terapia ocupacional en el adulto mayor con depresión" realizado por López y cols., en el 2017, donde se trabajó con un total de 31 Adultos Mayores, se observó que la mayoría eran mujeres con $67.7 \%$ y hombres solo un $32.3 \%$. En cuanto a la depresión en el presente estudio el $69.6 \%$ no presenta depresión donde la población con la que se trabajo fue de 56 Adultos Mayores. Encontrando similitud con su estudio donde el $83.9 \%$ no presenta depresión de un total de 31 Adultos Mayores (López-Nolasco, et al., 2018).

Por otra parte, Bolaños Sánchez, et al., reportan que la prevalencia de depresión fue de $18.1 \%$ en los adultos mayores costarricenses (Bolaños-Sánchez, 2015); menor al porcentaje encontrado en los adultos mayores de Tlahuelilpan, donde el 30\% mostraron depresión.

Tabla 1. Frecuencia y porcentaje de variables sociodemográficas de los adultos mayores del Centro Gerontológico Integral de Tlahuelilpan, Hidalgo, México, 2018.

\begin{tabular}{|c|c|c|c|}
\hline Variable & & $f$ & $\%$ \\
\hline \multirow[t]{2}{*}{ Género } & Masculino & 16 & 28.6 \\
\hline & Femenino & 40 & 71.4 \\
\hline \multirow{4}{*}{$\begin{array}{l}\text { Nivel } \\
\text { académico }\end{array}$} & Primaria & 37 & 66.1 \\
\hline & Secundaria & 12 & 21.4 \\
\hline & Preparatoria & 5 & 8.9 \\
\hline & Universidad & 2 & 3.6 \\
\hline \multirow[t]{4}{*}{ Estado civil } & Soltero & 3 & 5.4 \\
\hline & Casado & 39 & 69.6 \\
\hline & Viudo & 11 & 19.6 \\
\hline & Divorciado & 3 & 5.4 \\
\hline \multirow[t]{5}{*}{ Ocupación } & Hogar & 39 & 69.6 \\
\hline & Campo & 5 & 8.9 \\
\hline & Obrero & 6 & 10.7 \\
\hline & Chofer & 1 & 1.8 \\
\hline & Jubilado & 5 & 8.9 \\
\hline \multirow{3}{*}{$\begin{array}{l}\text { Beneficios de } \\
\text { gobierno }\end{array}$} & Prospera & 3 & 5.4 \\
\hline & 65 y más & 30 & 53.6 \\
\hline & Ninguno & 23 & 41.1 \\
\hline \multirow{4}{*}{$\begin{array}{l}\text { Afiliación a } \\
\text { seguro médico }\end{array}$} & Seguro popular & 27 & 48.2 \\
\hline & IMSS & 15 & 26.8 \\
\hline & ISSSTE & 11 & 19.6 \\
\hline & Ninguna & 3 & 5.4 \\
\hline \multirow[t]{5}{*}{ Enfermedades } & Hipertensión & 14 & 25.0 \\
\hline & Diabetes & 6 & 10.7 \\
\hline & Ninguno & 18 & 32.1 \\
\hline & Otra & 3 & 5.4 \\
\hline & Hipertensión/Diabetes & 15 & 26.8 \\
\hline \multirow{3}{*}{$\begin{array}{l}\text { Personas } \\
\text { cohabitantes }\end{array}$} & Pareja & 37 & 66.1 \\
\hline & Hijos & 13 & 23.2 \\
\hline & Solo & 6 & 10.7 \\
\hline \multirow[t]{2}{*}{ Casa propia } & Sí & 52 & 92.9 \\
\hline & No & 4 & 7.1 \\
\hline
\end{tabular}

Fuente: Cuestionario de datos sociodemográficos. $\quad n=56$ 
Tabla 2. Frecuencia y porcentaje del nivel de depresión en primera y segunda medición de los adultos mayores del Centro Gerontológico Integral de Tlahuelilpan, Hidalgo, México, 2018.

\section{Nivel de depresión}

\begin{tabular}{lll}
\cline { 2 - 3 } & $\mathrm{f}$ & $\%$ \\
\hline Normal & 39 & 69.6 \\
\hline Leve & 15 & 26.8 \\
\hline Severa & 2 & 3.6 \\
\hline Total & 56 & 100
\end{tabular}

Fuente: Instrumento Escala de Depresión Geriátrica $(G D S)$ de Yesavage. $n=56$

\section{Conclusiones}

La mayoría de los adultos mayores que asiste a las actividades programadas en un Centro Gerontológico obtuvo nivel de depresión normal, no obstante, una tercera parte calificó a depresión leve o severa, por lo que se sugiere establecer programa grupal que aborde tanatología, aumento de la autoestima, satisfacción con la vida, etc., así como una atención psicológica individualizada.

\section{Agradecimientos}

A las autoridades del Centro Gerontológico Integral de Tlahuelilpan por su apoyo a los adultos mayores participantes por su participación.

\section{Referencias}

Acosta-López A. (2016). Prevalencia de depresión en el adulto mayor en los centros de vida en Cartagena año 2015.Tesis de licenciatura. Universidad de Cartagena. Recuperado el 28 de abril de 2019:

http://190.242.62.234:8080/jspui/bitstream/11227/3098/1/P REVALENCIA DE DEPRESION EN LOS CENTROS DE VIDA DE CARTAGENA INFORME FINAL.pdf

Bolaños-Sánchez, M. \& Helo-Guzmán, F. (2015). Depresión en el adulto mayor costarricense y su relación con la autopercepción del estado de salud y desempeño. Revista Clínica de la Escuela de Medicina UCR-HSJD, 5(4), 78-87. Recuperado en 28 de abril de 2019, de https://doi.org/10.15517/rc_ucr-hsjd.v5i4.20777

CNDH. (2017). Emite CNDH informe especial sobre personas mayores en los centros penitenciarios de la República Mexicana. Recuperado el 28 de abril de 2019, de Comisión Nacional de los Derechos Humanos: http://www.cndh.org.mx/sites/all/doc/Comunicados/2017/Co m_2017_318.pdf

Durán-Badillo, T., Aguilar, R. M., Martínez, M. L., Rodríguez, T., Gutiérrez, G., \& Vázquez, L. (2013). Depresión y función cognitiva de adultos mayores de una comunidad urbano marginal. Enfermería universitaria, 10(2), 36-42. Recuperado en 28 de abril de 2019, de http://www.scielo.org.mx/scielo.php?script=sci_arttext\&pid $=$ S1665-70632013000200002\&lng=es\&tlng=es...

IMSS. (2014). Depresión en el Adulto Mayor. Recuperado el 28 de abril de 2019, de Instituto Mexicano del Seguro Social: http://www.imss.gob.mx/salud-en-linea/preguntas-desalud/depresion-adultomayor

López-Nolasco, B., Maya-Sánchez, A., Cano-Estrada, E.A., Pérez-Corona, R., Ortega-Pérez, M. \& Serrano-Sánchez, M.D. (2018). Efecto de la terapia ocupacional en el autoconcepto del adulto mayor con depresión. XIKUA Boletín Científico de la Escuela Superior de Tlahuelilpan, 6(12), 1-12. Recuperado el 28 de abril de 2019: https://doi.org/10.29057/xikua.v6i12.3209

OMS. (2017). Depresión: qué debe saber. Recuperado el 28 de abril de 2019, de Organización Mundial de la Salud: http://www.who.int/campaigns/world-healthday/2017/handouts-depression/what-you-should-know/es/

Secretaría de Salud. (2011). Diagnóstico y Tratamiento de la Depresión en el Adulto Mayor en el Primer Nivel de Atención. Recuperado el 28 de abril de 2019, de Secretaría de Salud: http://www.cenetec.salud.gob.mx/descargas/gpc/CatalogoM aestro/194_GPC_DEPRESION_AM/GPC_DEPRESION_A DULTO_1er_NIV.pdf 